\title{
One-Variable and Multi-Mariable Calculus on a Non-Archimedean Field Extension of the Real Numbers*
}

\author{
Khodr Shamseddine** \\ Department of Physics and Astronomy, University of Manitoba \\ Winnipeg, Manitoba R3T 2N2, Canada \\ Received August 29, 2012
}

\begin{abstract}
New elements of calculus on a complete real closed non-Archimedean field extension $\mathcal{F}$ of the real numbers $\mathbb{R}$ will be presented. It is known that the total disconnectedness of $\mathcal{F}$ in the topology induced by the order makes the usual (topological) notions of continuity and differentiability too weak to extend real calculus results to $\mathcal{F}$. In this paper, we introduce new stronger concepts of continuity and differentiability which we call derivate continuity and derivate differentiability [2, 12]; and we show that derivate continuous and differentiable functions satisfy the usual addition, product and composition rules and that $n$-times derivate differentiable functions satisfy a Taylor formula with remainder similar to that of the real case. Then we generalize the definitions of derivate continuity and derivate differentiability to multivariable $\mathcal{F}$-valued functions and we prove related results that are useful for doing analysis on $\mathcal{F}$ and $\mathcal{F}^{n}$ in general.
\end{abstract}

DOI: $10.1134 / \mathrm{S} 2070046613020040$

Key words: non-Archimedean analysis, complete valued fields, multivariate calculus, derivate differentiability, Taylor formula.

\section{INTRODUCTION}

We start this section by reviewing some basic terminology and facts about non-Archimedean fields. So let $F$ be an ordered non-Archimedean field extension of $\mathbb{R}$. We introduce the following terminology.

Definition $1.1\left(\sim, \approx, \ll, S_{F}, \lambda\right)$. For $x, y \in F^{*}:=F \backslash\{0\}$, we say $x \sim y$ if there exist $n, m \in \mathbb{N}$ such that $n|x|>|y|$ and $m|y|>|x|$, where $|\cdot|$ denotes the ordinary absolute value on $F$ :

$$
|x|= \begin{cases}x & \text { if } x \geq 0 \\ -x & \text { if } x<0\end{cases}
$$

For nonnegative $x, y \in F$, we say that $x$ is infinitely smaller than $y$ and write $x \ll y$ if $n x<y$ for all $n \in \mathbb{N}$, and we say that $x$ is infinitely small if $x \ll 1$ and $x$ is finite if $x \sim 1$; finally, we say that $x$ is approximately equal to $y$ and write $x \approx y$ if $x \sim y$ and $|x-y| \ll|x|$. We also set $\lambda(x)=[x]$, the class of $x$ under the equivalence relation $\sim$.

The set of equivalence classes $S_{F}$ (under the relation $\sim$ ) is naturally endowed with an addition via $[x]+[y]=[x \cdot y]$ and an order via $[x]<[y]$ if $|y| \ll|x|($ or $|x| \gg|y|)$, both of which are readily checked to be well-defined. Note that we use + instead of - for the operation in $S_{F}$ because, for the fields we will be interested in (see below), $S_{F}$ is isomorphic to an additive subgroup of $\mathbb{R}$. It follows that $\left(S_{F},+, \leq\right)$ is an ordered group, often referred to as the Hahn group or skeleton group, whose neutral element is [1], the class of 1 . It follows from the above that the projection $\lambda$ from $F^{*}$ to $S_{F}$ is a valuation.

The theorem of Hahn [3] provides a complete classification of non-Archimedean extensions of $\mathbb{R}$ in terms of their skeleton groups. In fact, invoking the axiom of choice it is shown that the elements of any

\footnotetext{
* The text was submitted by the author in English.

${ }^{* *}$ E-mail: khodr@physics . umanitoba.ca
} 
such ordered field $F$ can be written as (generalized) formal power series (also called Hahn series) over its skeleton group $S_{F}$ with real coefficients, and the set of appearing exponents forms a well-ordered subset of $S_{F}$. That is, for all $x \in F$, we have

$$
x=\sum_{q \in S_{F}} a_{q} d^{q}
$$

with $a_{q} \in \mathbb{R}$ for all $q, d$ a positive infinitely small element of $F$, and the support of $x$, given by

$$
\operatorname{supp}(x):=\left\{q \in S_{F}: a_{q} \neq 0\right\},
$$

forming a well-ordered subset of $S_{F}$. With the representation of elements of $F$ as in Equation (1.1) it follows that, for $x \neq 0$ in $F$,

$$
\lambda(x)=\min (\operatorname{supp}(x)),
$$

which exists since $\operatorname{supp}(x)$ is well-ordered. Moreover, we set $\lambda(0)=\infty$.

Addition, multiplication and order on the Hahn series are defined as follows. Given $x=\sum_{q \in \operatorname{supp}(x)} a_{q} d^{q}$ and $y=\sum_{t \in \operatorname{Supp}(y)} b_{t} d^{t}$, then

$$
\begin{aligned}
& x+y=\sum_{r \in \operatorname{supp}(x) \cup \operatorname{supp}(y)}\left(a_{r}+b_{r}\right) d^{r} ; \text { and } \\
& x \cdot y=\sum_{r \in \operatorname{supp}(x) \oplus \operatorname{supp}(y)}\left(\sum_{\substack{q \in \operatorname{supp}(x), t \in \operatorname{supp}(y) \\
q+t=r}} a_{q} \cdot b_{t}\right) d^{r} .
\end{aligned}
$$

Note that, $\operatorname{since} \operatorname{supp}(x)$ and $\operatorname{supp}(y)$ are well-ordered, only finitely many terms contribute to the sum

$$
\sum_{\substack{q \in \operatorname{supp}(x), t \in \operatorname{supp}(y) \\ q+t=r}} a_{q} \cdot b_{t},
$$

in Equation (1.2), for each $r \in \operatorname{supp}(x) \oplus \operatorname{supp}(y)$.

Given a nonzero $x=\sum_{q \in \operatorname{Supp}(x)} a_{q} d^{q}$, then $x>0$ if and only if $a_{\lambda(x)}>0$.

From general properties of formal power series fields [7,9], it follows that if $S_{F}$ is divisible then $F$ is real closed; that is, every positive element of $F$ is a square in $F$ and every polynomial of odd degree over $F$ has at least one root in $F$. For a general overview of the algebraic properties of formal power series fields, we refer to the comprehensive overview by Ribenboim [10], and for an overview of the related valuation theory the book by Krull [4]. A thorough and complete treatment of ordered structures can also be found in $[8]$.

Throughout this paper, we will denote by $\mathcal{F}$ any totally ordered non-Archimedean field extension of $\mathbb{R}$ that is real closed and complete in the order topology and whose skeleton group $S_{\mathcal{F}}$ is Archimedean, i.e. a subgroup of $\mathbb{R}$. The coefficient $a_{q}$ of the $q$ th power in the Hahn representation of a given $x$ will be denoted by $x[q]$, and hence the number $d$ is given by $d[1]=1$ and $d[q]=0$ for $q \neq 1$. It is easy to check that, for $q \in S_{\mathcal{F}}, 0<d^{q} \ll 1$ if and only if $q>0$, and $d^{q} \gg 1$ if and only if $q<0$; moreover, $x \approx x[\lambda(x)] d^{\lambda(x)}$ for all $x \neq 0$.

The smallest such field $\mathcal{F}$ is the Levi-Civita field $\mathcal{R}$, first introduced in [5, 6]. In this case $S_{\mathcal{R}}=\mathbb{Q}$, and for any element $x \in \mathcal{R}, \operatorname{supp}(x)$ is a left-finite subset of $\mathbb{Q}$, i.e. below any rational bound $r$ there are only finitely many exponents in the Hahn representation of $x$. The Levi-Civita field $\mathcal{R}$ is of particular interest because of its practical usefulness. Since the supports of the elements of $\mathcal{R}$ are left-finite, it is possible to represent these numbers on a computer [1]. Having infinitely small numbers, the errors in classical numerical methods can be made infinitely small and hence irrelevant in all practical applications. One 
such application is the computation of derivatives of real functions representable on a computer [14], where both the accuracy of formula manipulators and the speed of classical numerical methods are achieved. For a review of the Levi-Civita field $\mathcal{R}$, see [1, 12-23].

In the wider context of valuation theory, it is interesting to note that the topology induced by the order on $\mathcal{F}$ is the same as that introduced via the valuation $\lambda$, as shown in Remark 1.2 below. It follows therefore that the field $\mathcal{F}$ is just a special case of the class of fields discussed in [11].

Remark 1.2. The mapping $\Lambda: \mathcal{F} \times \mathcal{F} \rightarrow \mathbb{R}$, given by $\Lambda(x, y)=\exp (-\lambda(x-y))$, is an ultrametric distance (and hence a metric); the valuation topology it induces is equivalent to the order topology. For if $A$ is an open set in the order topology and $a \in A$, then there exists $r>0$ in $\mathcal{F}$ such that, for all $x \in \mathcal{F},|x-a|<r \Rightarrow x \in A$. Let $l=\exp (-\lambda(r))$, then we also have that, for all $x \in \mathcal{F}, \Lambda(x, a)<l \Rightarrow x \in A$; and hence $A$ is open with respect to the valuation topology. The other direction of the equivalence of the topologies follows analogously.

It follows from Remark 1.2 that $\mathcal{F}$ which is complete in the order topology is also complete in the valuation topology induced by the ultrametric $\Lambda$.

The total disconnectedness of the field $\mathcal{F}$ in the order topology leads to difficulties in doing analysis on the field. For example (see [12]), contrary to the real case, there are continuous functions on an interval $[a, b] \subset \mathcal{F}$ that do not satisfy the intermediate value theorem on $[a, b]$. Moreover, one can easily come up with a nonconstant function whose derivative is zero everywhere; and this makes integration much harder and renders the solutions of the simplest initial value problems not unique. One can also construct a function that is not increasing on an interval while its derivative is strictly positive on that interval as well as a function whose first non-vanishing derivative at $x_{0}$ of an open interval $] a, b[$ is positive and of even order while the function assumes a relative maximum at $x_{0}$; and this makes the study of optimization problems more difficult than in the real case. One approach to circumvent such difficulties, is to introduce stronger continuity and differentiability notions than the usual $\epsilon-\delta$ ones used in real analysis.

In this paper, we review the definitions of the so-called derivate continuity and differentiability in one dimension (first introduced in $[2,12]$ in the special case of $\mathcal{F}=\mathcal{R}$ ) and then we state and prove several related results, leading to the proof of Taylor formula with remainder as well as a discussion of the existence of Taylor series representation for an infinitely often derivate differentiable function. Then we generalize these notions of continuity and differentiability to higher dimensions, again proving that $m$-times derivate differentiable functions from an open subset of $\mathcal{F}^{n}$ to $\mathcal{F}$ satisfy a Taylor formula with remainder.

The fact that the derivate differentiability concept (but not the ordinary differentiability) leads to a Taylor formula with remainder for functions that are $m$-times derivate differentiable and to a Taylor series for functions that are infinitely often derivate differentiable, just as in real analysis, allows us to avoid some of the problems mentioned above that are caused by the disconnectedness of the field in the order topology. This is particularly useful, for example, for obtaining (unconstrained and constrained) optimization results similar to those from the real case; please see [22, 23] for the details.

\section{DERIVATE CONTINUITY AND DIFFERENTIABILITY}

Throughout this section, $I(a, b)$ will denote any one of the intervals $] a, b[] a, b,],[a, b[$ or $[a, b]$.

Definition 2.1. Let $a<b$ be given in $\mathcal{F}$ and let $f: I(a, b) \rightarrow \mathcal{F}$. Then we say that $f$ is derivate continuous on $I(a, b)$ if there exists $M \in \mathcal{F}$, called a Lipschitz constant of $f$ on $I(a, b)$, such that

$$
\left|\frac{f(y)-f(x)}{y-x}\right| \leq M \text { for all } x \neq y \text { in } I(a, b) .
$$

Note that the $|\cdot|$ in Definition 2.1 is the ordinary absolute value defined on $\mathcal{F}$ in the Introduction. It follows immediately from Definition 2.1 that if $f: I(a, b) \rightarrow \mathcal{F}$ is derivate continuous on $I(a, b)$ then $f$ is uniformly continuous and bounded on $I(a, b)$. 
Remark 2.2. It is clear that the concept of derivate continuity in Definition 2.1 coincides with that of Lipschitz continuity when restricted to $\mathbb{R}$. We chose to call it derivate continuity here so that, after having defined derivate differentiability in Definition 2.8 and higher order derivate differentiability in Definition 2.12, we can think of derivate continuity as derivate differentiability of "order zero", just as is the case for continuity in $\mathbb{R}$.

Remark 2.3. Definition 2.1 can be generalized in the obvious way to functions on any countable unions of intervals of $\mathcal{F}$.

Proposition 2.4. Let $a<b$ be given in $\mathcal{F}$, let $f, g: I(a, b) \rightarrow \mathcal{F}$ be derivate continuous on $I(a, b)$, and let $\alpha \in \mathcal{F}$. Then $f+\alpha g$ and $f \cdot g$ are derivate continuous on $I(a, b)$.

Proof. Since $f$ and $g$ are derivate continuous on $I(a, b)$, there exist $M_{1}, M_{2} \in \mathcal{F}$ such that

$$
\left|\frac{f(y)-f(x)}{y-x}\right| \leq M_{1} \text { and }\left|\frac{g(y)-g(x)}{y-x}\right| \leq M_{2} \text { for all } x \neq y \text { in } I(a, b) .
$$

Let

$$
M=\max \left\{M_{1}, M_{2}\right\}
$$

Then

$$
\left|\frac{f(y)-f(x)}{y-x}\right| \leq M \text { and }\left|\frac{g(y)-g(x)}{y-x}\right| \leq M \text { for all } x \neq y \text { in } I(a, b) .
$$

Now let $x \neq y$ in $I(a, b)$ be given. Then

$$
\begin{aligned}
\left|\frac{(f+\alpha g)(y)-(f+\alpha g)(x)}{y-x}\right| & =\left|\frac{f(y)+\alpha g(y)-f(x)-\alpha g(x)}{y-x}\right| \\
& \leq\left|\frac{f(y)-f(x)}{y-x}\right|+|\alpha|\left|\frac{g(y)-g(x)}{y-x}\right| \\
& \leq(1+|\alpha|) M .
\end{aligned}
$$

Hence $f+\alpha g$ is derivate continuous on $I(a, b)$ with Lipschitz constant $(1+|\alpha|) M$.

Since $f$ and $g$ are derivate continuous on $I(a, b)$, it follows that $f$ and $g$ are bounded on $I(a, b)$. Hence there exists $M_{0} \in \mathcal{F}$ such that

$$
|f(x)| \leq M_{0} \text { and }|g(x)| \leq M_{0} \text { for all } x \in I(a, b) .
$$

Now for all $x \neq y$ in $I(a, b)$, we have that

$$
\begin{aligned}
\left|\frac{(f \cdot g)(y)-(f \cdot g)(x)}{y-x}\right| & =\left|\frac{f(y) g(y)-f(x) g(x)}{y-x}\right| \\
& =\left|\frac{f(y)(g(y)-g(x))+(f(y)-f(x)) g(x)}{y-x}\right| \\
& \leq|f(y)|\left|\frac{g(y)-g(x)}{y-x}\right|+|g(x)| \frac{f(y)-f(x)}{y-x} \mid \\
& \leq M_{0} M_{2}+M_{0} M_{1} \\
& \leq 2 M_{0} M .
\end{aligned}
$$

Hence $f \cdot g$ is derivate continuous on $I(a, b)$ with Lipschitz constant $2 M_{0} M$.

Proposition 2.5. Let $a<b$ and $c<e$ in $\mathcal{F}$ be given, and let $f: I_{1}(a, b) \rightarrow \mathcal{F}$ and $g: I_{2}(c, e) \rightarrow \mathcal{F}$ be such that $f\left(I_{1}(a, b)\right) \subset I_{2}(c, e), f$ is derivate continuous on $I_{1}(a, b)$ and $g$ derivate continuous on $I_{2}(c, e)$. Then $g \circ f$ is derivate continuous on $I_{1}(a, b)$. 
Proof. Let $M_{f}$ and $M_{g}$ be Lipschitz constants of $f$ on $I_{1}(a, b)$ and of $g$ on $I_{2}(c, e)$, respectively. Let $x \neq y$ be given in $I_{1}(a, b)$. First assume that $f(y)=f(x)$. Then

$$
\left|\frac{(g \circ f)(y)-(g \circ f)(x)}{y-x}\right|=\left|\frac{g(f(y))-g(f(x))}{y-x}\right|=0 \leq M_{g} M_{f} .
$$

Now assume that $f(y) \neq f(x)$. Then

$$
\begin{aligned}
\left|\frac{(g \circ f)(y)-(g \circ f)(x)}{y-x}\right| & =\left|\frac{g(f(y))-g(f(x))}{y-x}\right| \\
& =\left|\frac{g(f(y))-g(f(x))}{f(y)-f(x)} \frac{f(y)-f(x)}{y-x}\right| \\
& =\left|\frac{g(f(y))-g(f(x))}{f(y)-f(x)}\right|\left|\frac{f(y)-f(x)}{y-x}\right| \\
& \leq M_{g} M_{f} .
\end{aligned}
$$

Thus, for all $y \neq x$ in $I_{1}(a, b)$, we have that

$$
\left|\frac{(g \circ f)(y)-(g \circ f)(x)}{y-x}\right| \leq M_{g} M_{f}
$$

and hence $(g \circ f)$ is derivate continuous on $I_{1}(a, b)$, with Lipschitz constant $M_{g} M_{f}$.

Proposition 2.6. Let $a<b$ be given in $\mathcal{F}$ and let $f: I(a, b) \rightarrow \mathcal{F}$ be derivate continuous on $I(a, b)$. Then there exists a unique function $g:[a, b] \rightarrow \mathcal{F}$, derivate continuous on $[a, b]$, such that $\left.g\right|_{I(a, b)}=f$.

Proof. We may assume that $I(a, b) \neq[a, b]$. First assume that $I(a, b)=] a, b]$. Let

$$
f_{0}=\lim _{n \rightarrow \infty} f\left(a+d^{n}(b-a)\right)
$$

where $d$ is the positive infinitely small number defined in the introduction. The limit exists in $\mathcal{F}$ [12] because $f$ is uniformly continuous on $] a, b]$ and $\mathcal{F}$ is complete. Define $g:[a, b] \rightarrow \mathcal{F}$ by $g(x)=f(x)$ if $x \in] a, b]$ and $g(a)=f_{0}$. It remains to show that $g$ is derivate continuous on $[a, b]$. Let $M$ be a Lipschitz constant of $f$ on $] a, b]$ and let $x \neq y$ in $[a, b]$ be given. Without loss of generality, we may assume that $x<y$. First assume that $a<x$, then $x, y \in] a, b]$, and hence

$$
\left|\frac{g(y)-g(x)}{y-x}\right|=\left|\frac{f(y)-f(x)}{y-x}\right| \leq M<2 M .
$$

Now assume that $x=a$. There exists $N \in \mathbb{N}$ such that $d^{N}(b-a)<y-a$ and

$$
\left|f\left(a+d^{N}(b-a)\right)-g(a)\right| \leq M(y-a) .
$$

Then it follows that $0<y-\left(a+d^{N}(b-a)\right)<y-a$; and hence

$$
\begin{aligned}
\left|\frac{g(y)-g(x)}{y-x}\right| & \leq\left|\frac{f(y)-f\left(a+d^{N}(b-a)\right)}{y-a}\right|+\left|\frac{f\left(a+d^{N}(b-a)\right)-g(a)}{y-a}\right| \\
& \leq\left|\frac{f(y)-f\left(a+d^{N}(b-a)\right)}{y-\left(a+d^{N}(b-a)\right)}\right|+M \\
& \leq M+M=2 M .
\end{aligned}
$$

Hence $g$ is derivate continuous on $[a, b]$ with Lipschitz constant $2 M$, and $\left.g\right|_{] a, b]}=f$.

Similarly, we can show that the result is true for the cases when $I(a, b)=[a, b[$ and $I(a, b)=] a, b[$.

Using an argument similar to that in the proof of Proposition 2.6, we obtain the following result. 
Corollary 2.7. Let $a<b$ be given in $\mathcal{F}$, let $x_{0} \in I(a, b)$, and let $f: I(a, b) \backslash\left\{x_{0}\right\} \rightarrow \mathcal{F}$ be derivate continuous on $I(a, b) \backslash\left\{x_{0}\right\}$. Then there exists a unique function $g: I(a, b) \rightarrow \mathcal{F}$, derivate continuous on $I(a, b)$, such that $\left.g\right|_{I(a, b) \backslash\left\{x_{0}\right\}}=f$.

Definition 2.8. Let $a<b$ be given in $\mathcal{F}$, let $f: I(a, b) \rightarrow \mathcal{F}$ be derivate continuous on $I(a, b)$, and let $I_{D}$ denote the identity function on $I(a, b)$. Then we say that $f$ is derivate differentiable on $I(a, b)$ if for all $x \in I(a, b)$, the function $\frac{f-f(x)}{I_{D}-x}: I(a, b) \backslash\{x\} \rightarrow \mathcal{F}$ is derivate continuous on $I(a, b) \backslash\{x\}$. In this case the unique continuation of $\frac{f-f(x)}{I_{D}-x}$ to $I(a, b)$, obtained by Corollary 2.7 , will be called the first derivate function (or simply the derivate function) of $f$ at $x$ and will be denoted by $F_{1, x}$; moreover, the function value $F_{1, x}(x)$ will be called the derivative of $f$ at $x$ and will be denoted by $f^{\prime}(x)$.

It follows immediately from Definition 2.8 that if $f: I(a, b) \rightarrow \mathcal{F}$ is derivate differentiable then $f$ is differentiable in the conventional sense; moreover, the two derivatives at any given point of $I(a, b)$ agree. As for derivate continuity, the definition of derivate differentiability can be generalized to functions on countable unions of intervals of $\mathcal{F}$.

Theorem 2.9. Let $a<b$ be given in $\mathcal{F}$, let $f, g: I(a, b) \rightarrow \mathcal{F}$ be derivate differentiable on $I(a, b)$, and let $\alpha \in \mathcal{F}$. Then $f+\alpha g$ and $f \cdot g$ are derivate differentiable on $I(a, b)$, with derivatives $(f+\alpha g)^{\prime}=f^{\prime}+\alpha g^{\prime}$ and $(f \cdot g)^{\prime}=f^{\prime} \cdot g+f \cdot g^{\prime}$.

Proof. Let $x \in I(a, b)$ be given, and let $F_{1, x}$ and $G_{1, x}$ denote the derivate functions of $f$ and $g$ at $x$, respectively. Then $F_{1, x}$ and $G_{1, x}$ are derivate continuous on $I(a, b)$. It follows by Proposition 2.4 that the function $F_{1, x}+\alpha G_{1, x}: I(a, b) \rightarrow \mathcal{F}$, given by

$$
\begin{aligned}
\left(F_{1, x}+\alpha G_{1, x}\right)(y) & =F_{1, x}(y)+\alpha G_{1, x}(y) \\
& = \begin{cases}\frac{f(y)+\alpha g(y)-f(x)-\alpha g(x)}{y-x} & \text { if } y \neq x \\
f^{\prime}(x)+\alpha g^{\prime}(x) & \text { if } y=x\end{cases} \\
& = \begin{cases}\frac{(f+\alpha g)(y)-(f+\alpha g)(x)}{y-x} & \text { if } y \neq x \\
\left(f^{\prime}+\alpha g^{\prime}\right)(x) & \text { if } y=x,\end{cases}
\end{aligned}
$$

is derivate continuous on $I(a, b)$. This is true for all $x \in I(a, b)$. Hence $f+\alpha g$ is derivate differentiable on $I(a, b)$ with derivative $(f+\alpha g)^{\prime}=f^{\prime}+\alpha g^{\prime}$.

Now let $x \in I(a, b)$ be given, and let $H_{x}: D \rightarrow \mathcal{F}$ be given by

$$
H_{x}(y)= \begin{cases}\frac{(f \cdot g)(y)-(f \cdot g)(x)}{y-x} & \text { if } y \neq x \\ f^{\prime}(x) g(x)+f(x) g^{\prime}(x) & \text { if } y=x .\end{cases}
$$

We show that $H_{x}$ is derivate continuous on $I(a, b)$ for all $x \in I(a, b)$. Note that

$$
H_{x}(y)= \begin{cases}\frac{f(y) g(y)-f(x) g(x)}{y-x} & \text { if } y \neq x \\ f^{\prime}(x) g(x)+f(x) g^{\prime}(x) & \text { if } y=x\end{cases}
$$




$$
\begin{aligned}
& = \begin{cases}\frac{f(y)-f(x)}{y-x} g(x)+f(y) \frac{g(y)-g(x)}{y-x} & \text { if } y \neq x \\
f^{\prime}(x) g(x)+f(x) g^{\prime}(x) & \text { if } y=x\end{cases} \\
& =F_{1, x}(y) g(x)+f(y) G_{1, x}(y) .
\end{aligned}
$$

Hence

$$
H_{x}=g(x) \cdot F_{1, x}+f \cdot G_{1, x} .
$$

Since $f, F_{1, x}$ and $G_{1, x}$ are derivate continuous on $I(a, b)$, so is $H_{x}$ by Proposition 2.4. This is true for all $x \in I(a, b)$, and hence $(f \cdot g)$ is derivate differentiable on $I(a, b)$, with derivative

$$
(f \cdot g)^{\prime}(x)=H_{x}(x)=f^{\prime}(x) g(x)+f(x) g^{\prime}(x)
$$

for all $x \in I(a, b)$.

Theorem 2.10 (Chain Rule). Let $a<b$ and $c<e$ in $\mathcal{F}$, and let $f: I_{1}(a, b) \rightarrow \mathcal{F}$ and $g: I_{2}(c, e) \rightarrow \mathcal{F}$ be such that $f\left(I_{1}(a, b)\right) \subset I_{2}(c, e), f$ is derivate differentiable on $I_{1}(a, b)$ and $g$ derivate differentiable on $I_{2}(c, e)$. Then $g \circ f$ is derivate differentiable on $I_{1}(a, b)$, with derivative $(g \circ f)^{\prime}=$ $\left(g^{\prime} \circ f\right) \cdot f^{\prime}$.

Proof. Let $x \in I_{1}(a, b)$ be given, and let $H_{x}: I_{1}(a, b) \rightarrow \mathcal{F}$ be given by

$$
H_{x}(y)= \begin{cases}\frac{(g \circ f)(y)-(g \circ f)(x)}{y-x} & \text { if } y \neq x \\ g^{\prime}(f(x)) f^{\prime}(x) & \text { if } y=x .\end{cases}
$$

Then

$$
\begin{aligned}
H_{x}(y) & = \begin{cases}\frac{g(f(y))-g(f(x))}{f(y)-f(x)} \frac{f(y)-f(x)}{y-x} & \text { if } y \neq x \text { and } f(y) \neq f(x) \\
0 & \text { if } y \neq x \text { and } f(y)=f(x) \\
g^{\prime}(f(x)) f^{\prime}(x) & \text { if } y=x\end{cases} \\
& =G_{1, f(x)}(f(y)) F_{1, x}(y),
\end{aligned}
$$

where $F_{1, x}$ is the derivate function of $f$ at $x$, and $G_{1, f(x)}$ the derivate function of $g$ at $f(x)$. Hence $H_{x}=$ $\left(G_{1, f(x)} \circ f\right) \cdot F_{1, x}$. Since $f$ is derivate continuous on $I_{1}(a, b)$ and since $G_{1, f(x)}$ is derivate continuous on $I_{2}(c, e)$, we have by Proposition 2.5 that $\left(G_{1, f(x)} \circ f\right)$ is derivate continuous on $I_{1}(a, b)$. Since $F_{1, x}$ is derivate continuous on $I_{1}(a, b)$, so is $\left(G_{1, f(x)} \circ f\right) \cdot F_{1, x}=H_{x}$ by Proposition 2.4. Hence $(g \circ f)$ is derivate differentiable on $I_{1}(a, b)$, with derivative

$$
(g \circ f)^{\prime}(x)=H_{x}(x)=g^{\prime}(f(x)) f^{\prime}(x)=\left(\left(g^{\prime} \circ f\right) \cdot f^{\prime}\right)(x)
$$

for all $x \in I_{1}(a, b)$.

The following result provides a useful tool for checking the derivate differentiability of functions.

Theorem 2.11. Let $a<b$ be given in $\mathcal{F}$ and let $f: I(a, b) \rightarrow \mathcal{F}$ be derivate continuous on $I(a, b)$. Suppose there exists $M \in \mathcal{F}$ and there exists a function $g: I(a, b) \rightarrow \mathcal{F}$ such that

$$
\left|\frac{f(y)-f(x)}{y-x}-g(x)\right| \leq M|y-x| \text { for all } y \neq x \text { in } I(a, b) .
$$

Then $f$ is derivate differentiable on $I(a, b)$, with derivative $f^{\prime}=g$. 
Proof. It suffices to show that for all $x \in I(a, b)$, the function $F_{1, x}: I(a, b) \rightarrow \mathcal{F}$, given by

$$
F_{1, x}(y)= \begin{cases}\frac{f(y)-f(x)}{y-x} & \text { if } y \neq x \\ g(x) & \text { if } y=x,\end{cases}
$$

is derivate continuous on $I(a, b)$. We will show that for all $x \in I(a, b)$, we have that $\left|F_{1, x}(y)-F_{1, x}(z)\right| \leq$ $d^{-1} M|y-z|$ for all $y \neq z$ in $I(a, b)$. So let $x \in I(a, b)$ be given; and let $y \neq z$ in $I(a, b)$. Four cases are to be considered.

Case 1: Assume $y=x$. Then

$$
\begin{aligned}
\left|F_{1, x}(y)-F_{1, x}(z)\right| & =\left|F_{1, x}(x)-F_{1, x}(z)\right|=\left|g(x)-\frac{f(z)-f(x)}{z-x}\right| \\
& \leq M|x-z|=M|y-z|<d^{-1} M|y-z| .
\end{aligned}
$$

Case 2: Assume $z=x$. As in case 1 , we have that $\left|F_{1, x}(y)-F_{1, x}(z)\right| \leq M|y-z|<d^{-1} M|y-z|$.

Case 3: Assume $y \neq x \neq z$ and $|y-z| \nless|y-x|$. Then $|y-z| \nless|z-x|$; for if $|y-z| \ll|z-x|$, then $|y-x|=|y-z+(z-x)| \approx|z-x| \gg|y-z|$, a contradiction. Thus,

$$
\begin{aligned}
\left|F_{1, x}(y)-F_{1, x}(z)\right| & \leq\left|\frac{f(y)-f(x)}{y-x}-g(x)\right|+\left|\frac{f(z)-f(x)}{z-x}-g(x)\right| \\
& \leq M|y-x|+M|z-x|<d^{-1} M|y-z|
\end{aligned}
$$

since $d^{-1}|y-z| \gg|y-x|+|z-x|$.

Case 4: Assume $y \neq x \neq z$ and $|y-z| \ll|y-x|$. Then $z-x=z-y+(y-x) \approx y-x$. Thus

$$
\begin{aligned}
& \left|F_{1, x}(y)-F_{1, x}(z)\right|=\left|\frac{f(y)-f(x)}{y-x}-\frac{f(z)-f(x)}{z-x}\right| \\
= & \left|\frac{y-z}{y-x}\right|\left|\frac{f(y)-f(z)}{y-z}-\frac{f(z)-f(x)}{z-x}\right| \\
\leq & \left|\frac{y-z}{y-x}\right|\left(\left|\frac{f(y)-f(z)}{y-z}-g(z)\right|+\left|\frac{f(z)-f(x)}{z-x}-g(z)\right|\right) \\
\leq & \left|\frac{y-z}{y-x}\right| M(|y-z|+|z-x|) .
\end{aligned}
$$

Since $|z-x| \approx|y-x|$, we obtain that $|z-x|<d^{-1}|y-x| / 2$. Also, since $|y-z| \ll|y-x|$, we obtain that $|y-z|<|y-x|<d^{-1}|y-x| / 2$. Therefore,

$$
\left|F_{1, x}(y)-F_{1, x}(z)\right| \leq\left|\frac{y-z}{y-x}\right| M d^{-1}|y-x|=d^{-1} M|y-z| .
$$

Definition 2.12 ( $n$-times Derivate Differentiability). Let $a<b$ be given in $\mathcal{F}$, and let $f: I(a, b) \rightarrow \mathcal{F}$. Let $n \geq 2$ be given in $\mathbb{N}$. Then we define $n$-times derivate differentiability of $f$ on $I(a, b)$ inductively as follows: Having defined $(n-1)$-times derivate differentiability, we say that $f$ is $n$-times derivate differentiable on $I(a, b)$ if $f$ is $(n-1)$-times derivate differentiable on $I(a, b)$ and for all $x \in I(a, b)$, the $(n-1)$ st derivate function $F_{n-1, x}$ is derivate differentiable on $I(a, b)$. For all $x \in I(a, b)$, the derivate function $F_{n, x}$ of $F_{n-1, x}$ at $x$ will be called the $n$th derivate function of $f$ at $x$, and the number $f^{(n)}(x)=n ! F_{n-1, x}^{\prime}(x)$ will be called the $n$th derivative of $f$ at $x$ and denoted by $f^{(n)}(x)$.

In connection with the derivate functions, we introduce the secants of different orders. 
Definition 2.13. Let $a<b$ be given in $\mathcal{F}$ and let $f: I(a, b) \rightarrow \mathcal{F}$. Then for all $x \in I(a, b)$, the function $S_{1, x}: I(a, b) \backslash\{x\} \rightarrow \mathcal{F}$, given by

$$
S_{1, x}(y)=\frac{f(y)-f(x)}{y-x}
$$

will be called the first secant of $f$ at $x$.

Definition 2.14. Let $a<b$ be given in $\mathcal{F}$, let $n \in \mathbb{N}$ be given and let $f: I(a, b) \rightarrow \mathcal{F}$ be $n$-times derivate differentiable on $I(a, b)$. Let $x \in I(a, b)$ be given, and let $F_{1, x}, F_{2, x}, \ldots$, and $F_{n, x}$ denote the first, second, ..., and $n$th derivate functions of $f$ at $x$, respectively. For all $l \in\{2, \ldots, n+1\}$, define $S_{l, x}: I(a, b) \backslash\{x\} \rightarrow \mathcal{F}$ to be the first secant of $F_{l-1, x}$ at $x$. Then $S_{l, x}$ will be called the $l$ th secant of $f$ at $x$.

Lemma 2.15. Let $n \in \mathbb{N}$ be given, let $a<b$ be given in $\mathcal{F}$, and let $f: I(a, b) \rightarrow \mathcal{F}$ be $n$-times derivate differentiable on $I(a, b)$. Let $f^{\prime}, \ldots, f^{(n)}$ denote the first, .., nth derivative functions of $f$ on $I(a, b)$, and for all $x \in I(a, b)$, let $F_{1, x}, \ldots, F_{n, x}$ denote the first, ..,nth derivate functions of $f$ at $x$. Then for all $x, y \in I(a, b)$, we have that

$$
\begin{aligned}
f(y) & =f(x)+F_{1, x}(y)(y-x) \\
& =f(x)+f^{\prime}(x)(y-x)+F_{2, x}(y)(y-x)^{2} \\
& \vdots \\
& =f(x)+\sum_{j=1}^{n-1} \frac{f^{(j)}(x)}{j !}(y-x)^{j}+F_{n, x}(y)(y-x)^{n} .
\end{aligned}
$$

Proof. We use induction on $n$. The assertion is true for $n=1$ by definition of $F_{1, x}$. We assume it is true for $n=l$ and show it is true for $n=l+1$. So let $f$ be $(l+1)$-times derivate differentiable on $I(a, b)$. Since $f$ is $l$-times derivate differentiable on $I(a, b)$, we have by the induction hypothesis that

$$
f(y)=f(x)+\sum_{j=1}^{l-1} \frac{f^{(j)}(x)}{j !}(y-x)^{j}+F_{l, x}(y)(y-x)^{l}
$$

for all $x, y \in I(a, b)$. Since $f$ is $(l+1)$-times derivate differentiable on $I(a, b)$, we have that $F_{l, x}$ is derivate differentiable on $I(a, b)$, with derivative

$$
F_{l, x}^{\prime}(x)=\frac{f^{(l+1)}(x)}{(l+1) !}
$$

and with derivate function $F_{l+1, x}$, the $(l+1)$ st derivate function of $f$ at $x$. Thus,

$$
\begin{aligned}
F_{l, x}(y) & =F_{l, x}(x)+F_{l+1, x}(y)(y-x) \\
& =F_{l-1, x}^{\prime}(x)+F_{l+1, x}(y)(y-x) \\
& =\frac{f^{(l)}(x)}{l !}+F_{l+1, x}(y)(y-x)
\end{aligned}
$$

for all $x, y \in I(a, b)$.

Substituting Equation (2.2) into Equation (2.1) yields

$$
f(y)=f(x)+\sum_{j=1}^{l} \frac{f^{(j)}(x)}{j !}(y-x)^{j}+F_{l+1, x}(y)(y-x)^{l+1}
$$

for all $x, y \in I(a, b)$. So the assertion is true for $n=l+1$; and hence it is true for all $n \in \mathbb{N}$. 
Corollary 2.16. Let $a<b$ be given in $\mathcal{F}$, let $n \in \mathbb{N}$ be given and let $f: I(a, b) \rightarrow \mathcal{F}$ be $n$-times derivate differentiable on $I(a, b)$. Then for all $l \in\{2, \ldots, n+1\}$ and for all $y \neq x$ in $I(a, b)$, we have that

$$
S_{l, x}(y)=\frac{f(y)-\sum_{j=0}^{l-1} \frac{f^{(j)}(x)}{j !}(y-x)^{j}}{(y-x)^{l}} .
$$

Proof. Let $y \neq x$ in $I(a, b)$ and $l \in\{2, \ldots, n+1\}$ be given. Then, using Lemma 2.15, we obtain that

$$
\begin{aligned}
S_{l, x}(y) & =\frac{F_{l-1, x}(y)-F_{l-1, x}(x)}{y-x} \\
& =\frac{1}{y-x}\left(\frac{f(y)-\sum_{j=0}^{l-2} \frac{f^{(j)}(x)}{j !}(y-x)^{j}}{(y-x)^{l-1}}-\frac{f^{(l-1)}(x)}{(l-1) !}\right) \\
& =\frac{f(y)-\sum_{j=0}^{l-1} \frac{f^{(j)}(x)}{j !}(y-x)^{j}}{(y-x)^{l}} .
\end{aligned}
$$

Corollary 2.17 (Taylor Formula with Remainder). Let $a<b$ be given in $\mathcal{F}$ and let $f: I(a, b) \rightarrow \mathcal{F}$ be n-times derivate differentiable on $I(a, b)$. Let $x \in I(a, b)$ be given, let $F_{n, x}$ be the nth order derivate function of $f$ at $x$, and let $M_{n, x}$ be a Lipschitz constant of $F_{n, x}$. Then for all $y \in I(a, b)$, we have that

$$
f(y)=f(x)+\sum_{j=1}^{n} \frac{f^{(j)}(x)}{j !}(y-x)^{j}+r_{n}(x, y)(y-x)^{n+1},
$$

with $\lambda\left(r_{n}(x, y)\right) \geq \lambda\left(M_{n, x}\right)$.

Proof. If $y=x$, there is nothing to prove; so we may assume that $y \neq x$. Since $F_{n, x}$ is derivate continuous on $I(a, b)$, with Lipschitz constant $M_{n, x}$, we have that

$$
F_{n, x}(y)=F_{n, x}(x)+r_{n}(x, y)(y-x), \text { with } \lambda\left(r_{n}(x, y)\right) \geq \lambda\left(M_{n, x}\right) .
$$

Using Lemma 2.15, we have that

$$
F_{n, x}(y)=\frac{f(y)-f(x)-\sum_{j=1}^{n-1} \frac{f^{(j)}(x)}{j !}(y-x)^{j}}{(y-x)^{n}} .
$$

Also, from Definition 2.12, we obtain that

$$
F_{n, x}(x)=F_{n-1, x}^{\prime}(x)=\frac{f^{(n)}(x)}{n !} .
$$

Thus,

$$
\frac{f(y)-f(x)-\sum_{j=1}^{n-1} \frac{f^{(j)}(x)}{j !}(y-x)^{j}}{(y-x)^{n}}=\frac{f^{(n)}(x)}{n !}+r_{n}(x, y)(y-x)
$$

and hence

$$
f(y)=f(x)+\sum_{j=1}^{n} \frac{f^{(j)}(x)}{j !}(y-x)^{j}+r_{n}(x, y)(y-x)^{n+1} .
$$


Theorem 2.18. Let $n \in \mathbb{N}$ be given; let $a<b$ be given in $\mathcal{R}$; let $f, g: I(a, b) \rightarrow \mathcal{F}$ be $n$-times derivate differentiable on $I(a, b)$, with derivatives $f^{\prime}, \ldots, f^{(n)}$ and $g^{\prime}, \ldots, g^{(n)}$, respectively; and let $\alpha \in \mathcal{F}$ be given. Then $f+\alpha g$ is $n$-times derivate differentiable on $I(a, b)$, with derivatives

$$
(f+\alpha g)^{(l)}=f^{(l)}+\alpha g^{(l)} \text { for all } l \in\{1, \ldots, n\} .
$$

Proof. We use induction on $n$. The assertion is true for $n=1$ by Theorem 2.9. We assume it is true for $n=m$ and show it is true for $n=m+1$. So we have that $f$ and $g$ are $(m+1)$-times derivate differentiable on $I(a, b)$. By the induction hypothesis, we have that $(f+\alpha g)$ is $m$-times derivate differentiable on $I(a, b)$ with derivatives

$$
(f+\alpha g)^{(l)}=f^{(l)}+\alpha g^{(l)} \text { for all } l \in\{1, \ldots, m\} .
$$

Now let $x \in I(a, b)$ be given. Since $f$ and $g$ are $(m+1)$-times derivate differentiable on $I(a, b)$, we have that the $(m+1)$ st derivate functions of $f$ and $g$ at $x, F_{m+1, x}, G_{m+1, x}: I(a, b) \rightarrow \mathcal{F}$, given by

$$
F_{m+1, x}(y)= \begin{cases}\frac{f(y)-f(x)-\sum_{l=1}^{m} \frac{f^{(l)}(x)}{l !}(y-x)^{l}}{(y-x)^{m+1}} & \text { if } y \neq x \\ \frac{f^{(m+1)}(x)}{(m+1) !} & \text { if } y=x\end{cases}
$$

and

$$
G_{m+1, x}(y)= \begin{cases}\frac{g(y)-g(x)-\sum_{l=1}^{m} \frac{g^{(l)}(x)}{l !}(y-x)^{l}}{(y-x)^{m+1}} & \text { if } y \neq x \\ \frac{g^{(m+1)}(x)}{(m+1) !} & \text { if } y=x,\end{cases}
$$

are derivate continuous on $I(a, b)$. By Proposition 2.4, we have that $F_{m+1, x}+\alpha G_{m+1, x}: I(a, b) \rightarrow \mathcal{F}$, given by

$$
\begin{aligned}
& \left(F_{m+1, x}+\alpha G_{m+1, x}\right)(y)=F_{m+1, x}(y)+\alpha G_{m+1, x}(y) \\
= & \begin{cases}\frac{f(y)+\alpha g(y)-(f(x)+\alpha g(x))-\sum_{l=1}^{m} \frac{f^{(l)}(x)+\alpha g^{(l)}(x)}{l !}(y-x)^{l}}{(y-x)^{m+1}} & \text { if } y \neq x \\
\frac{f^{(m+1)}(x)+\alpha g^{(m+1)}(x)}{(m+1) !} & \text { if } y=x\end{cases} \\
= & \begin{cases}\frac{(f+\alpha g)(y)-(f+\alpha g)(x)-\sum_{l=1}^{m} \frac{(f+\alpha g)(l)}{l !}(x)}{(y-x)^{m+1}(y-x)^{l}} & \text { if } y \neq x \\
\frac{f^{(m+1)}(x)+\alpha g^{(m+1)}(x)}{(m+1) !} & \text { if } y=x,\end{cases}
\end{aligned}
$$

is derivate continuous on $I(a, b)$, where use has been made of Equation (2.3). Hence $f+\alpha g$ is $(m+1)$ times derivate differentiable on $I(a, b)$, with $(m+1)$ st derivative

$$
(f+\alpha g)^{(m+1)}(x)=f^{(m+1)}(x)+\alpha g^{(m+1)}(x) \text { for all } x \in I(a, b) .
$$

So the assertion is true for $n=m+1$, and hence it is true for all $n \in \mathbb{N}$.

Definition 2.19. Let $a<b$ be given in $\mathcal{F}$, and let $f: I(a, b) \rightarrow \mathcal{F}$. Then we say that $f$ is infinitely often derivate differentiable on $I(a, b)$ if for all $n \in \mathbb{N}, f$ is $n$-times derivate differentiable on $I(a, b)$.

Using Theorem 3.3 in [15], we obtain the following result. 
Theorem 2.20. Let $a<b$ be given in $\mathcal{F}$, and let $f: I(a, b) \rightarrow \mathcal{F}$ be infinitely often derivate differentiable on $I(a, b)$. Let $x_{0} \in I(a, b)$ be given, and let

$$
\lambda_{0}=\limsup _{n \rightarrow \infty}\left(\frac{-\lambda\left(f^{(n)}\left(x_{0}\right)\right)}{n}\right) .
$$

Then $\sum_{n=0}^{\infty} f^{(n)}\left(x_{0}\right) /(n !)\left(x-x_{0}\right)^{n}$ converges (in the order topology) if $\lambda\left(x-x_{0}\right)>\lambda_{0}$, and it is divergent if $\lambda\left(x-x_{0}\right)<\lambda_{0}$.

The following example shows that, even when

$$
\sum_{n=0}^{\infty} \frac{f^{(n)}\left(x_{0}\right)}{n !}\left(x-x_{0}\right)^{n}
$$

converges, the series need not converge to $f(x)$. It converges to $f(x)$ only if the remainder term $r_{n}\left(x_{0}, x\right)\left(x-x_{0}\right)^{n+1}$ converges to 0 ; and Theorem 2.21 below provides a criterion for that.

Example 2.1. Let $f:[-1,1] \rightarrow \mathcal{F}$ be given by

$$
f(x)= \begin{cases}\exp \left(-1 / x^{2}\right) & \text { if } x \sim 1 \\ 0 & \text { if } 0 \leq|x| \ll 1\end{cases}
$$

Then $f$ is infinitely often derivate differentiable on $[-1,1]$, and we have that

$$
f^{(n)}(0)=0 \text { for all } n \geq 1 .
$$

Thus,

$$
\sum_{n=0}^{\infty} \frac{f^{(n)}(0)}{n !} x^{n} \text { converges to } 0 \text { for all } x \in[-1,1] .
$$

Hence the limit is equal to $f(x)$ if $0 \leq|x| \ll 1$ and is different from $f(x)$ if $x \sim 1$.

Theorem 2.21. Let $a<b$ be given in $\mathcal{F}$ and let $f: I(a, b) \rightarrow \mathcal{F}$ be infinitely often derivate differentiable on $I(a, b)$. Let $x_{0} \in I(a, b)$ be given and for each $l \in \mathbb{N}$, let $F_{l, x_{0}}$ denote the lth derivate function of $f$ at $x_{0}$. For each $l \in \mathbb{N}$, let

$$
\alpha_{l}=\sup _{\text {in } \mathbb{R}}\left\{\lambda\left(M_{l}\right): M_{l} \text { is a Lipschitz constant of } F_{l, x_{0}}\right\},
$$

and let

$$
\lambda_{0}=\limsup _{l \rightarrow \infty}\left(\frac{-\alpha_{l}}{l}\right) .
$$

Then $\sum_{n=0}^{\infty} f^{(n)}\left(x_{0}\right) /(n !)\left(x-x_{0}\right)^{n}$ converges to $f(x)$ for all $x \in I(a, b)$ satisfying

$$
\lambda\left(x-x_{0}\right)>\lambda_{0}
$$

Proof. Let $x \in I(a, b)$ be such that $\lambda\left(x-x_{0}\right)>\lambda_{0}$ and let $l \in \mathbb{N}$ be given. By Corollary 2.17, we have that

$$
f(x)=\sum_{n=0}^{l} \frac{f^{(n)}\left(x_{0}\right)}{n !}\left(x-x_{0}\right)^{n}+r_{l}\left(x_{0}, x\right)\left(x-x_{0}\right)^{l+1},
$$

where

$$
\lambda\left(r_{l}\left(x_{0}, x\right)\right) \geq \lambda\left(M_{l}\right)
$$


for any Lipschitz constant $M_{l}$ of $F_{l, x_{0}}$; and hence

$$
\lambda\left(r_{l}\left(x_{0}, x\right)\right) \geq \alpha_{l} .
$$

We need to show that

$$
\lim _{l \rightarrow \infty}\left(r_{l}\left(x_{0}, x\right)\left(x-x_{0}\right)^{l+1}\right)=0 .
$$

Since $\lambda\left(x-x_{0}\right)>\lambda_{0}$, there exists $t \in \mathbb{Q}^{+}$such that

$$
\lambda\left(x-x_{0}\right)-t>\lambda_{0} .
$$

Hence there exists $N \in \mathbb{N}$ such that

$$
\lambda\left(x-x_{0}\right)-t>\frac{-\alpha_{l}}{l} \text { for all } l \geq N .
$$

Hence

$$
\alpha_{l}+l \lambda\left(x-x_{0}\right)>l t \text { for all } l \geq N .
$$

Thus,

$$
\lambda\left(r_{l}\left(x_{0}, x\right)\left(x-x_{0}\right)^{l}\right)>l t \text { for all } l \geq N
$$

which entails that

$$
\lim _{l \rightarrow \infty}\left(r_{l}\left(x_{0}, x\right)\left(x-x_{0}\right)^{l}\right)=0
$$

Hence

$$
\lim _{l \rightarrow \infty}\left(r_{l}\left(x_{0}, x\right)\left(x-x_{0}\right)^{l+1}\right)=\left(x-x_{0}\right) \lim _{l \rightarrow \infty}\left(r_{l}\left(x_{0}, x\right)\left(x-x_{0}\right)^{l}\right)=0 .
$$

\section{DERIVATE CONTINUITY AND DERIVATE DIFFERENTIABILITY FOR MULTIVARIABLE FUNCTIONS}

Now we generalize the concepts of derivate continuity and derivate differentiability to functions of many variables. In the following, column vectors in $\mathcal{F}^{n}$ will be denoted by $\mathbf{x}, \mathbf{y}, \ldots$; and row vectors by $\mathbf{x}^{T}, \mathbf{y}^{T}, \ldots \mathcal{F}^{n}$ will be equipped with the product topology induced by the absolute value $|\cdot|: \mathcal{F}^{n} \rightarrow \mathcal{F}$, given by

$$
|\mathbf{x}|=\sqrt{x_{1}^{2}+x_{2}^{2}+\cdots+x_{n}^{2}}
$$

which is well-defined since $\mathcal{F}$ is real closed and hence roots of positive elements exist in $\mathcal{F}$. Note that, for any nonzero $\mathbf{x} \in \mathcal{F}^{n}$, we have that $\mathbf{u}=\mathbf{x} /|\mathbf{x}|$ is a unit vector in the sense that $|\mathbf{u}|=1$.

Definition 3.1. Let $D \subset \mathcal{F}^{n}$ be open, let $f: D \rightarrow \mathcal{F}$, and let $\mathbf{u}$ be a unit vector. For each $\mathbf{x} \in D$, let

$$
D_{\mathbf{x}, \mathbf{u}}=\{t \in \mathcal{F}: \mathbf{x}+t \mathbf{u} \in D\}
$$

and define $\phi_{\mathbf{x}, \mathbf{u}}: D_{\mathbf{x}, \mathbf{u}} \rightarrow \mathcal{F}$ by

$$
\phi_{\mathbf{x}, \mathbf{u}}(t)=f(\mathbf{x}+t \mathbf{u}) .
$$

Then we say that $f$ is derivate differentiable on $D$ in the direction of $\mathbf{u}$ if $\phi_{\mathbf{x}, \mathbf{u}}$ is derivate differentiable on $D_{\mathbf{x}, \mathbf{u}}$ for all $\mathbf{x}$ in $D$. Moreover, the derivative $\phi_{\mathbf{x}, \mathbf{u}}^{\prime}(0)$ will be called the directional derivative of $f$ at $\mathbf{x}$ in the $\mathbf{u}$ direction and will be denoted by $\partial_{\mathbf{u}} f(\mathbf{x})$.

Definition 3.2 (Partial Derivatives). Let $D \subset \mathcal{F}^{n}$ be open, let $f: D \rightarrow \mathcal{F}$ and let $\left\{\mathbf{e}_{1}, \ldots, \mathbf{e}_{n}\right\}$ denote the standard orthonormal basis of $\mathcal{F}^{n}$. Then the partial derivatives of $f$ are defined as the directional derivatives of $f$ in the directions $\mathbf{e}_{1}, \ldots, \mathbf{e}_{n}$, if these exist. If the partial derivative in the direction $\mathbf{e}_{k}$ exists, we will denote it by $\partial_{k} f$. The gradient of $f$, denoted by $\nabla f$, is defined to be the row vector whose components are the (first order) partial derivatives of $f$. 
Definition 3.3. Let $D \subset \mathcal{F}^{n}$ be open, let $f: D \rightarrow \mathcal{F}$ and let $q \in \mathbb{N}$ be given. Then we say that $f$ is $C^{q}$ on $D$ if all the partial derivatives of order smaller than or equal to $q$ exist and are derivate continuous on D.

Theorem 3.4. Let $D \subset \mathcal{F}^{n}$ be open, let $f: D \rightarrow \mathcal{F}$ be $C^{1}$ on $D$ and let $\mathbf{x} \in D$ be given. Then there exist $\delta, M>0$ in $\mathcal{F}$ such that $B_{\delta}(\mathbf{x})=\left\{\mathbf{z} \in \mathcal{F}^{n}:|\mathbf{z}-\mathbf{x}|<\delta\right\} \subset D$, and $|f(\mathbf{y})-f(\mathbf{x})-\nabla f(\mathbf{x})(\mathbf{y}-\mathbf{x})|$ $\leq M|\mathbf{y}-\mathbf{x}|^{2}$ for all $\mathbf{y} \in B_{\delta}(\mathbf{x})$.

Proof. We use induction on the number of variables $n$. The result is true for $n=1$ by definition of derivate differentiability in one dimension. We assume it is true for $n=k$ and we show that it is true for $n=k+1$. So let $f: D \subset \mathcal{F}^{k+1} \rightarrow \mathcal{F}$ be $C^{1}$, and let $\mathbf{x} \in D$ be given. Since $D$ is open, there exists $\delta_{0}>0$ in $\mathcal{F}$ such that $B_{\delta_{0}}^{(k+1)}(\mathbf{x}) \subset D$. Write $\mathbf{x}=\left(x_{1}, \ldots, x_{k}, x_{k+1}\right)$, let $\zeta=\left(x_{1}, \ldots, x_{k}\right) \in \mathcal{F}^{k}$ and let

$$
D_{1}=\left\{\left(z_{1}, \ldots, z_{k}\right) \in \mathcal{F}^{k}:\left(z_{1}, \ldots, z_{k}, x_{k+1}\right) \in B_{\delta_{0}}^{(k+1)}(\mathbf{x})\right\} .
$$

Then $D_{1}=B_{\delta_{0}}^{(k)}(\zeta)$; and hence $D_{1}$ is open in $\mathcal{F}^{k}$. Define $\psi: D_{1} \rightarrow \mathcal{F}$ by

$$
\psi\left(z_{1}, \ldots, z_{k}\right)=f\left(z_{1}, \ldots, z_{k}, x_{k+1}\right) .
$$

Then $\psi$ is $C^{1}$ on $D_{1}$ and the partial derivatives of $\psi$ at $\left(z_{1}, \ldots, z_{k}\right)$ are given by

$$
\partial_{j} \psi\left(z_{1}, \ldots, z_{k}\right)=\partial_{j} f\left(z_{1}, \ldots, z_{k}, x_{k+1}\right) \text { for all } j \in\{1, \ldots, k\} \text {. }
$$

Thus, by the induction hypothesis, there exist $\delta_{1}, M_{1}>0$ in $\mathcal{F}$ such that $B_{\delta_{1}}^{(k)}(\zeta) \subset D_{1}$ (i.e. $\left.\delta_{1} \leq \delta_{0}\right)$ and

$$
|\psi(\eta)-\psi(\zeta)-\nabla \psi(\zeta)(\eta-\zeta)| \leq M_{1}|\eta-\zeta|^{2} \text { for all } \eta \in B_{\delta_{1}}^{(k)}(\zeta) .
$$

Let $\mathbf{y} \in B_{\delta_{1}}^{(k+1)}(\mathbf{x})$ and let $\mathbf{w}=\left(y_{1}, \ldots, y_{k}\right)$. Then $\mathbf{w} \in B_{\delta_{1}}^{(k)}(\zeta)$; and hence

$$
\begin{aligned}
& |f(\mathbf{y})-f(\mathbf{x})-\nabla f(\mathbf{x})(\mathbf{y}-\mathbf{x})| \\
\leq & \left|f(\mathbf{y})-\psi(\mathbf{w})-\partial_{k+1} f(\mathbf{x})\left(y_{k+1}-x_{k+1}\right)\right| \\
& +\left|\psi(\mathbf{w})-\psi(\zeta)-\sum_{j=1}^{k} \partial_{j} f(\mathbf{x})\left(y_{j}-x_{j}\right)\right| \\
= & \left|f\left(\mathbf{w}, y_{k+1}\right)-f\left(\mathbf{w}, x_{k+1}\right)-\partial_{k+1} f(\mathbf{x})\left(y_{k+1}-x_{k+1}\right)\right| \\
& +|\psi(\mathbf{w})-\psi(\zeta)-\nabla \psi(\zeta)(\mathbf{w}-\zeta)| \\
\leq & \left|f\left(\mathbf{w}, y_{k+1}\right)-f\left(\mathbf{w}, x_{k+1}\right)-\partial_{k+1} f\left(\mathbf{w}, x_{k+1}\right)\left(y_{k+1}-x_{k+1}\right)\right| \\
& +\left|\partial_{k+1} f\left(\mathbf{w}, x_{k+1}\right)-\partial_{k+1} f(\mathbf{x})\right|\left|y_{k+1}-x_{k+1}\right| \\
& +|\psi(\mathbf{w})-\psi(\zeta)-\nabla \psi(\zeta)(\mathbf{w}-\zeta)| \\
\leq & M_{2}\left|y_{k+1}-x_{k+1}\right|^{2}+M_{3}|\mathbf{w}-\zeta|\left|y_{k+1}-x_{k+1}\right|+M_{1}|\mathbf{w}-\zeta|^{2}
\end{aligned}
$$

for some constants $M_{2}, M_{3} \in \mathcal{F}$, which do not depend on $\mathbf{y}$ (nor on $\mathbf{x}$ ), since $\partial_{k+1} f$ is derivate continuous on $D$. Let $M=\max \left\{3 M_{1}, 3 M_{2}, 3 M_{3}\right\}$. Then

$$
\begin{aligned}
|f(\mathbf{y})-f(\mathbf{x})-\nabla f(\mathbf{x})(\mathbf{y}-\mathbf{x})| & \leq M_{2}|\mathbf{y}-\mathbf{x}|^{2}+M_{3}|\mathbf{y}-\mathbf{x}|^{2}+M_{1}|\mathbf{y}-\mathbf{x}|^{2} \\
& \leq M|\mathbf{y}-\mathbf{x}|^{2} .
\end{aligned}
$$

Thus, the result is true for $n=k+1$; and hence it is true for all $n$.

As an immediate consequence of Theorem 3.4, we obtain the following result.

Corollary 3.5. Let $D \subset \mathcal{F}^{n}$ be open, let $\mathbf{x}_{0} \in D$ be given and let $f: D \rightarrow \mathcal{F}$ be $C^{1}$ on $D$. Then there exist $M, \delta>0$ in $\mathcal{F}$ such that $B_{\delta}\left(\mathbf{x}_{0}\right) \subset D$ and, for all $\mathbf{x} \in B_{\delta}\left(\mathbf{x}_{0}\right)$, we have that

$$
f(\mathbf{x})=f\left(\mathbf{x}_{0}\right)+\nabla f\left(\mathbf{x}_{0}\right)\left(\mathbf{x}-\mathbf{x}_{0}\right)+R_{2}\left(\mathbf{x}_{0}, \mathbf{x}\right),
$$

where $\left|R_{2}\left(\mathbf{x}_{0}, \mathbf{x}\right)\right| \leq M\left|\mathbf{x}-\mathbf{x}_{0}\right|^{2}$. 
Lemma 3.6. Let $D \subset \mathcal{F}^{n}$ be open, and let $f: D \rightarrow \mathcal{F}$ be $C^{1}$ on $D$. Fix $\mathbf{x}_{0} \in D$, let $\mathbf{v} \in \mathcal{F}^{n}$ be a fixed vector and let $D_{1} \subset \mathcal{F}$ be given by $D_{1}=\left\{t \in \mathcal{F}: \mathbf{x}_{0}+t \mathbf{v} \in D\right\}$. Define $\phi: D_{1} \rightarrow \mathcal{F}$ by $\phi(t)=f\left(\mathbf{x}_{0}+t \mathbf{v}\right)$. Then $\phi$ is differentiable at 0 (in the conventional sense) with derivative $\phi^{\prime}(0)=$ $\nabla f\left(\mathbf{x}_{0}\right) \mathbf{v}$. Moreover, there exists $\delta>0$ in $\mathcal{F}$ such that $B_{\delta}(0) \subset D_{1}$ and

$$
\left|\frac{\phi(t)-\phi(0)}{t}-\phi^{\prime}(0)\right| \leq M|\mathbf{v}|^{2}|t| \text { for all } t \in B_{\delta}(0) \backslash\{0\} .
$$

Proof. By Theorem 3.4, there exist $\delta_{0}, M>0$ in $\mathcal{F}$ such that $B_{\delta_{0}}\left(\mathbf{x}_{0}\right) \subset D$ and

$$
\left|f(\mathbf{x})-f\left(\mathbf{x}_{0}\right)-\nabla f\left(\mathbf{x}_{0}\right)\left(\mathbf{x}-\mathbf{x}_{0}\right)\right| \leq M\left|\mathbf{x}-\mathbf{x}_{0}\right|^{2} \text { for all } \mathbf{x} \in B_{\delta_{0}}\left(\mathbf{x}_{0}\right) .
$$

Let $\delta=\delta_{0} /|\mathbf{v}|$. Then, since $B_{\delta_{0}}\left(\mathbf{x}_{0}\right) \subset D$, we obtain that $B_{\delta}(0) \subset D_{1}$. Now let $t \in B_{\delta}(0) \backslash\{0\}$ be given. Then $\mathbf{x}_{0}+t \mathbf{v} \in B_{\delta_{0}}\left(\mathbf{x}_{0}\right)$; and hence

$$
\left|f\left(\mathbf{x}_{0}+t \mathbf{v}\right)-f\left(\mathbf{x}_{0}\right)-\nabla f\left(\mathbf{x}_{0}\right) \mathbf{v} t\right| \leq M|\mathbf{v}|^{2} t^{2} .
$$

Thus,

$$
\left|\frac{\phi(t)-\phi(0)}{t}-\nabla f\left(\mathbf{x}_{0}\right) \mathbf{v}\right| \leq M|\mathbf{v}|^{2}|t|
$$

which finishes the proof of the lemma.

By repeated application of Corollary 3.5 and of Lemma 3.6 to the function $\phi$ and its derivatives, we obtain a Taylor formula with remainder, similar to the corresponding one in real calculus.

Corollary 3.7. [Taylor Formula for Functions of Several Variables / Let $D \subset \mathcal{F}^{n}$ be open, let $\mathbf{x}_{0} \in$ $D$ be given and let $f: D \rightarrow \mathcal{F}$ be $C^{q}$ on $D$. Then there exist $M, \delta>0$ in $\mathcal{F}$ such that $B_{\delta}\left(\mathbf{x}_{0}\right) \subset D$ and, for all $\mathbf{x} \in B_{\delta}\left(\mathbf{x}_{0}\right)$, we have that

$$
f(\mathbf{x})=f\left(\mathbf{x}_{0}\right)+\sum_{j=1}^{q}\left(\frac{1}{j !} \sum_{l_{1}, \ldots, l_{j}=1}^{n}\left(\partial_{l_{1}} \cdots \partial_{l_{j}} f\left(\mathbf{x}_{0}\right) \pi_{k=1}^{j}\left(x_{l_{k}}-x_{0, l_{k}}\right)\right)\right)+R_{q+1}\left(\mathbf{x}_{0}, \mathbf{x}\right),
$$

where $\left|R_{q+1}\left(\mathbf{x}_{0}, \mathbf{x}\right)\right| \leq M\left|\mathbf{x}-\mathbf{x}_{0}\right|^{q+1}$.

\section{ACKNOWLEDGMENTS}

I would like to recognize the contribution of Prof. Martin Berz from Michigan State University in the early stages of the development of this work. Discussions with Prof. Berz during my PhD studies led to the idea of derivate continuity and derivate differentiability; and then all of the results in one dimension included in this paper were developed in my PhD thesis [12] (in the special case of $\mathcal{F}=\mathcal{R}$ ). The multidimensional case presented in this paper was developed later as it was needed for the study of constrained optimization problems over $\mathcal{F}$ [23].

Moreover, I would like to thank the anonymous referee for his/her useful comments and suggestions on the initial version of the paper, which were incorporated in this final version.

\section{REFERENCES}

1. M. Berz, "Calculus and numerics on Levi-Civita fields," in M. Berz, C. Bischof, G. Corliss, and A. Griewank, editors, Computational Differentiation: Techniques, Applications, and Tools, pp. 19-35 (SIAM, Philadelphia, 1996).

2. M. Berz, "Nonarchimedean analysis and rigorous computation," Int. J. Appl. Math. 2, 889-930 (2000).

3. H. Hahn, "Über die nichtarchimedischen Größensysteme," Sitzungsbericht der Wiener Akademie der Wissenschaften Abt. 2a 117,601-655 (1907).

4. W. Krull, "Allgemeine Bewertungstheorie," J. Reine Angew. Math. 167, 160-196 (1932).

5. T. Levi-Civita, "Sugli infiniti ed infinitesimi attuali quali elementi analitici," Atti Ist. Veneto di Sc., Lett. ed Art. 7a (4), 1765 (1892).

6. T. Levi-Civita, “Sui numeri transfiniti,” Rend. Acc. Lincei 5a, 7 (91), 113 (1898). 
7. S. MacLane, "The universality of formal power series fields," Bull. Amer. Math. Soc. 45, 888 (1939).

8. S. Priess-Crampe, Angeordnete Strukturen: Gruppen, Körper, Projektive Ebenen (Springer, Berlin, 1983).

9. F. J. Rayner, "Algebraically closed fields analogous to fields of Puiseux series," J. London Math. Soc. 8, 504506 (1974).

10. P. Ribenboim, "Fields: algebraically closed and others," Manuscripta Math. 75, 115-150 (1992).

11. W. H. Schikhof, Ultrametric Calculus: An Introduction to p-Adic Analysis (Cambridge Univ. Press, 1985).

12. K. Shamseddine, New Elements of Analysis on the Levi-Civita Field, PhD thesis (Michigan State Univ., East Lansing, Michigan, USA, 1999), also Michigan State Univ. Report MSUCL-1147.

13. K. Shamseddine, "On the topological structure of the Levi-Civita field," J. Math. Anal. Appl. 368, 281-292 (2010).

14. K. Shamseddine and M. Berz, "Exception handling in derivative computation with non-Archimedean calculus," in M. Berz, C. Bischof, G. Corliss and A. Griewank, editors, Computational Differentiation: Techniques, Applications, and Tools, pp. 37-51 (SIAM, Philadelphia, 1996).

15. K. Shamseddine and M. Berz, "Convergence on the Levi-Civita field and study of power series," Proc. Sixth International Conference on p-Adic Functional Analysis, pp. 283-299 (Marcel Dekker, New York, 2000)

16. K. Shamseddine and M. Berz, "The differential algebraic structure of the Levi-Civita field and applications," Int. J. Appl. Math. 3, 449-465 (2000).

17. K. Shamseddine and M. Berz, "Intermediate values and inverse functions on non-Archimedean fields," Int. J. Math. Math. Sci. 30, 165-176 (2002).

18. K. Shamseddine and M. Berz, "Measure theory and integration on the Levi-Civita field," Contemp. Math. 319, 369-387 (2003).

19. K. Shamseddine and M. Berz, "Analytical properties of power series on Levi-Civita fields," Ann. Math. Blaise Pascal 12 (2), 309-329 (2005).

20. K. Shamseddine and M. Berz, "Generalized power series on a non-Archimedean field," Indag. Math. (N.S.) 17 (3), 457-477 (2006).

21. K. Shamseddine and M. Berz, "Intermediate value theorem for analytic functions on a Levi-Civita field," Bull. Belg. Math. Soc. Simon Stevin 14, 1001-1015 (2007).

22. K. Shamseddine and V. Zeidan, "One-dimensional optimization on non-Archimedean fields," J. Nonlinear Convex Anal. 2, 351-361 (2001).

23. K. Shamseddine and V. Zeidan, "Constrained second order optimization on non-Archimedean fields," Indag. Math. (N.S.) 14, 81-101 (2003). 